\title{
Phase Change Biological Switch: A New Hypothesis on the Mechanism of Cancer Formation
}

\author{
G Di Donna, MD, PhD* and R. Di Muro, MD
}

Brain Research Center, Italy

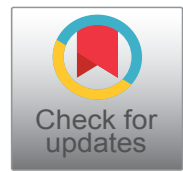

*Corresponding author: Giuseppe Di Donna, Brain Research Center, Via dei Cappellari 29, 00186 Rome, Italy

\begin{abstract}
The Authors hypothesize that, when in the presence of a chronic inflammatory process, the organism, while attempting to solve it, goes through a mechanism that the Authors define as "Phase Change Biological Switch": the cell line regression, which differentiated in that organ with the purpose of reprogramming to overcome the chronic inflammatory process, but regression involves loss of connections with the surrounding tissues and provokes the cancerous degeneration.

The importance of this new hypothesis is in the fact that it draws attention of research towards identifying that particular factor that we called "Phase Change Biological Switch", which turns a normal cell into a cancerous one. This "Phase Change Biological Switch", when reverted to its starting position, would start the whole set of factors leading the differentiation process; due to the fact that this process is partly unknown, the hypothesis about the "Phase Change Biological Switch", would go beyond the existing experimentation. This experimentation introduced only few factors (known factors, P53, iPARP, etc...) and got only a partial reduction of the tumor mass, both in laboratory and through clinical trials on different types of tumor.
\end{abstract}

\section{Definition of Cancer}

Cancer is a term indicating a disease in which abnormal cells divide without control and can invade nearby tissues ( $\mathrm{NCl}$ Dictionary of Cancer Terms).

Cancer is the name of a group of more than 100 diseases in which cells begin to grow out of control (American Cancer Society).

In this theoretical-experimental work, we particularly refer to the cancerous cell activity [1] not as a part of a specific tissue, but generically, also considering that the formulated hypothesis concerns the cell final degeneration and particularly the cell progressive evolution, from zygote to morula [2], through the totipotent, multipotent and oligopotent phase, up to the final differentiation [3] which turns a totipotent cell into a cancerous one [4].

\section{Attacking and Destroying Cancer}

Every natural event cannot prescind from nature itself and its working schemes [5]. That is to say that, in case of cancer pathology, nature behaves in such a way: "reacts to an external or internal stimulus of the organism itself by modifying the cellular structure in order to adapt it to changed conditions".

Inflammation plays an essential role in cell transformation processes: The inflammatory process is at the base of every mutation.

Inflammation intervenes in all reparative mechanism, and constitutes, or better includes, the biological switch which regulates cell transformation processes [6]. The genetic mechanism heads up the beginning evolution of the single originating cell, its partition [7] and differentiation in cell lines and in the further development of vessels, organs and apparatuses. The first step of research about cancer causes, has to start from these issues [8].

Cellular adaptation phenomena are common in nature and they are not classified as pathological events but as species evolution ones.

Starting from these considerations we can see how important is facing "cancer disease" from a different point of view, completely opposed to the present one, which, being affected by the "antibiotic mentality" 
wants to individuate a substance, with the aim of selectively destroying that specific tumor cell, as the antibiogram identifies targeted antibiotic therapy.

\section{Role of Inflammation}

The inflammation process is a phenomenon whose presence in nature constitutes the basis of every reparation process of organisms. The inflammation response, mediated by lymphocytes, is a response to an underway pathologic process [9]. Every injury, loss of substance, are lesions of the organism cured through a preliminary inflammatory response, which moves the defense mechanisms, activates the reparative processes, and causes a modification in the cellular elements [10].

Through these modifications every single cell obtains a reparative capacity.

The prerequisite of certain cancerous tissue modifications is often an inflammatory process which reacts to a noxa that can be internal or external to the organism.

The induction of transformation from normal tissue into a cancerous one, under the hypothesis put forward by the Authors, cannot be considered a pathological phenomenon, but a natural process which is activated by the organism to adapt the functions of the organ to the changed chemical, physical, hormonal, environmental conditions, internal or external to the organism itself, in order to survive.

The actual state of the art in oncology research is deeply affected by the influence which the actual antibiotic era has on medical scientific thinking.

The state of the art of cancer treatment consists basically on early diagnosis and on total eradication of the affected part. Similarly, therapeutic treatments of tumor mass ablation, in presence of metastatic lesions, need radiotherapy or chemotherapy, are giving results which often do not bring to a definitive solution.

Up to date tendencies, which consider the introduction of substances that can be selectively captured from cancer cells, with the aim of their selective and focused destruction, reveal a parasurgical therapeutic approach, which is far from the real objectives of the process which is run by the organism in order to protect and guarantee its own existence.

The large amount of studies in cancer treatment, which has been going on for many years, is enough to demonstrate that the general meaning of "cancer matter", except for some few types, such as leukemia, in which is operated a total marrow transplant, is still serious and unresolved; various therapies used until now, both pharmacological and radiotherapeutic ones, have proved to be palliative. Medicine seems to be powerless, giving no answers but the ones given by the enormous economic interests involved in this pathology [11].
There is no need of numbers, quotes or literary examples, to scientifically point out a reality which is not an opinion of the Authors but finds its validation on mortality statistics.

Publications and works concerning cancer research, just point out the importance and the therapeutic limit of the specific research, but there is nothing appearing to be the solution to this problem, which is still unsolved.

As an alternative, the health authorities just recommend prevention in order to early intervene, using the only effective therapy, which is the surgical excision, eventually extended, provided that there are no metastasis.

Undoubtedly, genetics and molecular research about cancer [12], which was pushed for the urgent need of having a solution, got excellent results by discovering determining factors in the differentiation process [13]. On the basis of these recent acquisitions, our hypothesis finds its research reason: It essentially suggests to leave the idea of selective cell disruption and, starting from the consideration (which we consider very important) that this evolutionary process towards cell carcinogenic phase must be interpreted not as a degenerative process but as a natural evolutionary phenomenon concerning cell adaptation to the adjacent microenvironment, it leads to the observation that the cell is able to redifferentiate into a normal one [14].

Essentially is a matter of inducing nature itself to trigger this redifferentiation process by using and controlling all the cellular complex mechanisms [15].

\section{Oncologic Immunotherapy}

Current research on the treatment of some cancers through oncologic immunotherapy [16], marks an important advance in changing the mentality: Finally, we are looking beyond the coarse concept of chemo and radiotherapy practiced as extreme and useless (when even harmful) therapeutic practices.

We begin to realize that only by managing natural defense mechanisms it is possible to face a problem belonging to the organism [17].

Beyond the possible developments of this method and it's still uncertain results [18], the timid awareness of trying to use the same mechanisms of nature's protection, remains fundamental, strengthening a new type of therapeutic orientation [19].

\section{Research Hypothesis: The Phase Change Bio- logical Switch}

The Authors hypothesize the presence, in the context of cellular differentiation process, of a genetic factor or a protein which is able to lead in an organized way, a process of adult cell differentiation, using the 
same properties which differentiated the cell into a unipotent [20] one having all the characteristics of the organ: This process goes through all the phases from the unipotent cell to the cancerous oligopotent one [21].

Essentially the Authors hypothesize that, when in the presence of a persistent endogenous (chemical/physical) or exogenous (hormonal, etc...) stimulus, the adult stem cells, using the same properties which have differentiated it from an oligopotent cell into a unipotent one, while remaining in the context of its tissue characteristic, through what the Authors define as "Phase Change Biological Switch" [22], in the natural and non-pathological attempt to readapt to the changed conditions of the surrounding microenvironment, partially reprograms itself into a cell which, even maintaining the "trace" of its originating tissue, takes on a phase (state) of potential reprogrammability [23], which is enough to make it lose the normal genetic relations with the mature cellular context in which it is found. Essentially, it is a state of immaturity which facilitates its uncontrolled growth, (cancerous stem cell CSc), even with its own originating tissue markings [24].

The definition, adopted by the Authors, of "Phase Change Switch" and in particular the term "Phase", to define the presence of a factor that intervenes in the transformation process of a stem cell from a normal cell to a cancer stem one (CSc) indicates what was hypothesized by the Authors themselves: The possibility of bringing back to the initial mature phase the immature cell (which the Authors do not consider cancerous) using, in a figurative way, an instrument capable of being switched in the two directions, inverse and reverse, to change the "phase" (state) of immaturity in which the cell finds itself.

This definition, which is more appropriately applied to a physical phenomenon rather than a biological one (it could be defined as a "state change factor"), was deliberately adopted by the Authors to highlight a concept which, even though currently ignored, is the cornerstone of their hypothesis: The cancer stem cell is actually the result, not of a pathological process, but of a natural evolution with the purpose of re-adaptation to mutated conditions of the surrounding microenvironment in order to reprogram itself to overcome the noxa (chronic inflammatory process, physical, chemical or hormonal stimulus etc.) that was the cause.

To clarify this concept, we cite an example in the field of physics from which the definition is borrowed.

Water can change phase and become ice or steam both with opposite properties: The phase change switch is the temperature which can increase or decrease. But we cannot say that ice or steam is the pathology of water.

\section{Stem Cells and Normal Cells}

Stem cells are primitive, non-specialized cells that have the ability to turn into different other types of cells through a process called cell differentiation.

Taken from different sources (umbilical cord, amniotic sac, bone marrow, blood, skin) they have some distinctive features:

a) Self-renewal through two types of division, obligatory asymmetric (the stem cell originates another stem cell and a cell destined to differentiate) and symmetric (stochastic differentiation of cells destined to differentiate) [25].

b) The pluripotency, which is the ability to originate one or more cell lines through differentiation and trans-differentiation (ability to change one's own cellular line modifying its development program).

The differentiation power of stem cell is highlighted through the various stages which is able to cover, from the initial state of totipotency (zygote) to the formation of the morula, to the state of pluripotency (in which it differentiates into endoderm, mesoderm, ectoderm) and progressively differentiating into cells called progenitors.

Subsequently, the differentiation process passes through the oligopotent stage to end with the ability to differentiate into a single type of cell (unipotency) called precursor cell.

A characteristic of the stem cell is that it can remain indefinitely in a state of quiescence as an undifferentiated cell and, if the conditions allow it, to be able to replicate itself in a symmetrical manner typical of the embryonic state (increasing stem cells) and in an adult phase, dividing into an asymmetrical form producing two cells: A specialized one destined to differentiate and an undifferentiated stem cell.

In the context of tissues, the ability to reproduce varies depending on the presence, in that tissue, of labile stem cells (always in active proliferation), stable (reduced proliferative capacity) and perennial (interrupted repair or replacement activity) (nervous system cells).

\section{Stem Cells and Epigenetic Code}

The ability of stem cells to differentiate responds to a precise logic that is exercising control over reproduction mechanisms in order to guarantee the correct regeneration of new tissue cells, to eliminate defective and deteriorated ones, bringing them to death.

Likewise, the possibility of inducing a stem cell to regress along the path of its differentiation into a unipotent stem cell and a mature one, has already been proven [26].

Experimental tests were in the past carried out in 
this sense: They were aimed at testing this regression property, both in the laboratory and through clinical trials on different types of tumors with results, however only partial, limited to the reduction of the tumor mass, but without reaching the eradication of the tumor and his metastases.

This therapeutic path, as shown by results, even if they are partial, is probably the right path that has served to identify and understand the importance of epigenetics as an operational tool of the genetic code, identifying many of the factors which intervene in both the differentiation process and the undifferentiation one, blocking the reproduction of the stem cell in the waiting phase (latent), and many other properties, such as the ability to make itself invisible to the killer cells of the immune system, through the PARP enzyme [27].

In this context, the attempt to use some of these factors to epigenetically interfere in the metabolism of the stem cell has proved, only partially valid, for three main factors:

1) The discovery of the epigenetic code that regulates the function of the genetic code is relatively recent and we currently do not have a complete "Use and Maintenance Manual".

2) Identifying these factors, such as P53 and PARP, to use them in differentiation processes is beyond their functional logic, since the stem regression has different, and very precise, aims, focused to ensure a correct control over cell division phases and the role of stemcells [28].

3) We just have a partial knowledge of epigenetic factors that intervene in cell division, the action of genes and their activation, the interaction between them and the microenvironment, or the synthesis of other proteins [29]. Ultimately the whole complex mechanism put in place and the balances between the various forces are unknown to us, so it is extremely complex to be able to drive from the outside a system that is programmed for different purposes than those we intend to obtain [30]. This explains why previous attempts to induce carcinogenic stem cell regression (CSc) have given results of only partial reduction of the tumor mass [31].

From these assumptions, we start our hypothesis: inducing the organism to autonomously complete the entire regression path by addressing the object of the research not to the single factors but to what we hypothesized to exist and that we have called "Phase Change Switch" (probably a protein).

\section{Phase Change Switch}

The hypothesis put forward by the Authors is that the organism, while acknowledging information about the chronicity level of the lesion and the inadequacy of the reparative processes, activates a sort of "Phase-
Change Biological Switch" which is suitable to allow the cell to go back over the initial process of differentiation [32], with the aim of making all the modifications which are suitable to adapt to the evolutionary phenomenon of environmental adaptation [33].

But this adaptation takes long time (many generations: see thalassemia).

In the case of cancer, the time which is necessary to the adaptation process and to the needed gene mutations relative to it, is much shorter if compared with the individual average lifespan, and can bring it to an and rather than to the pursued adaptation [34].

In this perspective, it is easy to understand how, even if absurdly, the definition of cancer as a disease does not have any foundation if analyzed with the perspective of nature which is directed to species conservation through the activation of the hypothesized "Phase Change Biological Switch"

\section{Switch of Phase Change Process}

We know that a stimulus, which is protracted for a certain period of time, originates a temporary modification in the cell. This modification, under the persistent stimulus, can originate a permanent modification, which can be genetically transmitted.

This observation introduces a concept, which maybe is not new, but we believe it is essential: The surprisingly cell plasticity at the molecular level [35].

The cell, analyzed as a functional independent complex, reproduces in an infinitely small settings, the same schema of the whole macroscopic cellular set [36]. In the single cell we find the same functional logic of the complex organism intended as an organized multicellular set. This concept can be easily and logically understood, because we are speaking of a set of functions reproducing a demultiplied specific function of every single cell [37]. In this way, if the differentiation process translated into a highly specific function of the single cell and if such cell has plastic capacity [38], if it is intimately modifiable thanks to biological switches which can interfere with the normal cell process of modifying DNA and responding to the new needs (internal or external stimuli) [39]. We have to individuate such biological switches and to find a way to activate or inactivate them, leading the phase transition process.

\section{Which is the nature of such Phase Switches? Pro- teins?}

We can hypothesize a biological molecular mechanism as an essential part of cell evolutive process, maybe a residual of that evolutionary process (memory?) which has remained latent but capable of being reactivated when occurring a chemical, biological physical stimulus having adequate intensity and persistence.

Cell modification involves DNA, and such a modifi- 
cation [40] (which can also be induced by a virus) has the aim of originating a cell regression phenomenon along of the evolutive differentiation path in order to reprogram the cell itself to make it compatible with the stimulus. Undifferentiation, regression, reprogrammation phenomena happen together with cell division phenomena and, at this step, activate biological switch function, which reprograms the two new cells originated by a stimulated mother cell [41].

Let us notice that in nervous system (= nervous cell) cellular regression phenomenon does not happen, because the neuron is a perennial element not subject to cell division (nervous system cancer does not exist). A persistent stimulus (conditioning) is however able to produce a modification of the nervous cell; this modification can be genetically transmitted when the stimulus (conditioning) became persistent enough [42].

The cell, which during the cell division has seen its DNA modified together with interconnection links with the neighboring cells, progressively unfastens from the function executed from the organ of which the cell itself is a part, therefore, the cell follows an independent reproduction path according to the new modified DNA schema, giving origin to a tumor mass which, progressively, induces compression or altered substitution of the function of the organ in which it is located [43].

\section{Cell Neoplasia and Phase Transition}

Correlation between cell neoplasia and Phase Transition outlines a common mechanism which justifies the assumption that is the object of this paper: "cancer is not a disease".

Let us make a consideration at the molecular level: We do not speak of physical, chemical or biological state, but we speak of molecular switch. Crossing of molecular elements is the property which differentiates physical switch from a chemical or a biological one.

The energetic characteristic of a biological switch is very different from the one of a chemical or physical switch, because energy, which has developed from its function intervenes as a response to an internal or external stimulus in a precise moment which is the one in which cell division occurs, and we would say that is closely correlated with cell division or even it is an essential part of it; that is to say that the biological switch should be intended to be a protein leading the division itself, because the possible DNA modification happens exactly at this stage.

This fact demonstrates that the organism, exposed to any stimulus, such a physical, chemical, biological one, always reacts with a biological response which is typical of a target organ using, as a phase transition biological switch, the process which started the cell division. Cell division is a process activated by cell ageing, which responses with precise parameters that transmit accurately the characteristics of the cell during chromosomes cross over; the secret of biological switch operation is in early stage of cell line differentiation of a development process realizing one way precise schemas which maintain the memory of every single stage of the evolution differentiation process.

Maybe, we can analyze one interpretative key of the two phenomena, tumor differentiation and cell division, within the stem cells context; stem cell is the only cell remaining totipotent [44]. Within a highly complex and organized system, which continually operates 24 hours a day, a complex check up of every single function, maintaining them under control and intervening to correct possible altered parameters, providing for possible reparation, are operations which are necessary after altered parameters are precisely and promptly identified. Organism individuates with absolute precision every alteration, lesion or damage, and promptly activates all the necessary reparation processes.

In the same way, with the objective of individual preservation, the organism provides to realize all the structural changes which better permit it to adapt to the changed environmental conditions [45]. This adaptation process has to consider as a physiological consequence which is proper of the organism. Such a plastic capability requests an obliged path which goes through the slow selection process which can operate necessary mutations within useful periods of time.

Generational selection means genetic modifications, which goes through paths that select stronger individuals which are able to generate new strong individuals. All these considerations explain the initial axiom "cancer is not a disease" and, as a consequence, they justify the orientation towards a different research line.

\section{Research Orientation}

The current research in the field of the epigenetic code has led to the identification of many of the factors that regulate the process of differentiation; some of these, as mentioned above, have been used in an attempt to induce a cellular regression, but being cellular regression a process of differentiation and regression that involves not a single factor, but more elements, it is essential to find that gene that coordinates these factors which are known today, and others that we do not yet know, completely controlling the whole differentiation process.

The aim of the research is therefore to identify, in the context of that "use and maintenance manual", which is the epigenetic code, the paragraph that describes the usage of the "switch" which is the regulator of the differentiation process.

The Authors recognize that this type of research requires a common commitment and that the present 
work, being a theoretical-experimental work, has the only limited objective of exposing a new hypothesis in order to identify that particular "switch" that is not able to block or stimulate that single gene but pilot the whole complex system of factors that regulate differentiation and stem plasticity.

Experience teaches that often, a scientific discovery happens because someone hypothesized its existence a priori.

\section{References}

1. AJ Becker, EA McCulloch, JE Till (1963) Cytological demonstration of the clonal nature of spleen colonies derived from transplanted mouse marrow cells. Nature 197: 452-454.

2. T Aoi, $\mathrm{K}$ Yae, M Nakagawa, T Ychisaka, K Okita, et al. (2008) Generation of pluripotent stem cells from adult mouse liver and stomach cells. Science 321: 699-702.

3. N Maherali, R Sridharan, W Xie, J Utikal, S Eminli, et al. (2007) Directly reprogrammed fibroblasts show global epigenetic reprogramming and widesprea tissue contribution Cell Stem Cell 1: 55-70.

4. M Wernig, A Meissner, R Foreman, T Brambrink, M Ku, et al. (2007) In vitro reprogramming of fibroblasts into a pluripotent ES-cell-like state Nature 448: 318-324.

5. S Mitalipov, D Wolf (2009) Totipotency, pluripotency and nuclear reprogramming. Adv Biochem Eng Biotecnol 114: 185-199.

6. James Byrne (2011) The definition and etymology of the word pluripotency e. Journal of Cellular Biotechnology 1: e2.

7. K Okita, T Ichisaka, S Yamanaka (2007) Generation of germline-competent induced pluripotent cells. Nature 448: 313-317.

8. K Takahashi, K Tanabe, M Ohnuki, M Narita, T Ichisaka, et al. (2007) Induction of pluripotent stem cells from adult human fibroblasts by defined factors. Cell 131: 861-872.

9. M Stadfield, N Maherali, DT Breault, K Hokedlinger (2008) Defining molecular cornerstones during fibroblast to IPS cell reprogramming in mouse. Cell Stem Cell 2: 230-240.

10. C Lois, EJ Hong, S Pease, EJ Brown, D Baltimore (2002) Germline transmission and tissue-specific expression of transgenes delivered by lentiviral vectors. Science 295 : 868-872.

11. J Hanna, S Markoulaki, P Schordrect, BW Carey, C Beard, et al. (2008) Direct reprogramming of terminally differentiated mature B lymphocytes to pluripotency. Cell 133: 250264.

12. BT Than, CY Park, LE Ailles, LL Weissman (2006) The cancer stem cells hypothesis a work in progress. Laboratory investigation 86: 1203-1207.

13. JS Lowengrub, HB Frieboes, F Jin, Y-L Chuang, $X \mathrm{Li}$, et al. (2010) Nonlinear modeling of cancer: Bridging the gap between cells and tumors. Nonlinearity 23: R1-R9.

14. K Abernaty, J Burke (2016) Modeling the treatment of glioblastoma multiforme and cancer stem cells with ordinary differential equations. Computational and Mathematical Methods in Medicine 2016: 1239861.

15. NL Komarowa (2013) Spatial stochastic models of cancer: fitness, migration, invasion. Mathematical Biosciences and Engineering 10: 761-775.
16. B Jones, RG Dale (1996) The reduction of tumor control with increasing overall time: Mathematical considerations. Br J Radiol 69: 830-838.

17. T Sakamaky, M Miyanishy (2019) Significance and purification of long-term hematopoietic stem cells in the hematopoietic system. Rinsho Ketsueki 60: 1056-1062.

18. S Galland, I Stamenkovic (2019) Mesenchymal stromal cells in cancer. A review of their immunomodulatory functions and dual effects on tumor progression. J Pathol.

19. P Zang, J Baxter, K Vinod, TN Tulenko, PJ Di Muzio (2009) Endothelial differentiation of amniotic fluid derived stem cells synergism of biochemical and shear force stimuli. Stem Cells Dev 8: 1299-1308.

20. N Siegel, A Valli, C Fuchs, M Rosner, M Henstschlager (2009) Induction of mesenchymal epithelial marker expression in human amniotic fluid stem cells Report. Biomed Online 19: 838-846.

21. M Takhahashi, S Yamanaa (2006) Induction of pluripotent stem cells from mouse embryonic and adult fibroblast cultures by defined factors. Cell 126: 663-676.

22. G Di Donna, R Di Muro (2014) Cancer disease: A new hypothesis of interpretation and therapeutic approach. Clin Med Rev Case Rep 1: 1-5.

23. M Nakagawa, S Yamanaka (2010) Reprogramming of somatic cells to pluripotency. The Cell Biology of Stem Cells 695: 215-224.

24. Yu J, Vodyanik MA, Smuga-Otto K, Antosiewicz-Bourget J, Frane JL, et al. (2007) Induced pluripotent stem cell lines derived from human somatic cells. Science 318: 19171920.

25. Varlakhanova NV, Cotterman RF, deVries WN, Morgan J, Donahue LR, et al. (2010) Myc maintains embrionic stem cell pluripotency and self-renewal. Differentiation 80: 9-19.

26. Zwaka TP, Thomson JA (2011) Homologus recombination in human embryonic stem cells. Nature Biotechnology 21: 319-321.

27. S Boussios, P Karihtala, M Moschetta, A Karathanasi, Sadauskaite, et al. (2019) Combined strategies with Poly (ADP-Ribose) Polymerase (PARP) inhibitors for the treatment of ovarian cancer: A literature review. Diagnostic (Basel) 9: E87.

28. E Borazanci, R Korn, WS Liang, C Guarnieri, S Haag, et al. (2019) An analysis of patients with DNA repair pathway mutations treated with a PARP inhibitor. Oncologist.

29. S Li, XY Li, TJ Zhang, MO Kamara, JVV Liang, et al. (2019) Design, synthesis and biological evaluation of homoerythina alkaloid derivatives bearing a triazole moiety as PARP-1 inhibitors and as potential antitumor drugs. Bioorg Chem, 103385.

30. Y Ji, Q Wang, Q Zhao, L Li, G Sun, et al. (2019) Autophagy suppression enhances DNA damage and cell death upon treatment with PARP Inhibitor Niraparib in laryngeal squamous cell carcinoma. Appl Microbiol Biotechnol.

31. SM Vairaven, MB Heemskerk, JL Lie, RM Barge, JJ Cornelissen, et al. (2005) The importance of identifying a backup donor for unrelated stem transplantation. Bone Marrow Transplant 35: 437-440.

32. M Lacroix, R Riscal, G Arena, LK Linares, L Le Cam (2019) Metabolic functions of tumor suppressor p53: implication in physiology, metabolic disorders, and cancer. Mol Metab S2212-8778: 30921-30924.

33. Fu X, Wu S, Li B, Xu Y, Liu J (2019) Functions of p53 in pluripotent stem cells. Protein Cell. 
34. ME Spaety, A Gries, A Badle, A Venkatasamy, B Romain, et al. (2019) HDAC4 levels control sensibility toward Csiplatin in gastric cancer via the p53p73 /BIK Pathway. Cancers (Basel) 11: E1747.

35. B Goldenson, JD Crispino (2015) The aurora kineases I cell cycle and leukemia. Oncogene 34: 537-545.

36. M Yan, C Wang, B He, M Yang, M Tong, et al. (2016) Aurora-A kinease: $A$ potent oncogene and target for cancer therapy. Med Rev 36: 1036-1079.

37. A Tang, K Gao, L Chu, R Zhang, J Jang, et al. (2017) Aurora kineases: Novel therapy targets in cancers. Oncotarget 8: 23937-23954.

38. T Kanagasabal, T Venkatesan, U Natarajan, S Alobid, K Alhazzani, et al. (2019) Regulation of cell cycle by MDM2 in prostate cancer cells through Aurora Kinease-B and p21WAF1 /CIP1 mediated pathways. Cell Signal 66: 109435.

39. J Meterns, MC Marchetto, C Bardi, FH Gage (2016) Evaluating cell reprogramming, differentiation and conversion technologies in uroscience. Nat Rev Neurosci 17: 424-437.
40. Y Wang, Y Bi, S Gao (2017) Epigenetic regulation of somatic cell reprogramming. Curr Opin Genet Dev 46: 156-163.

41. L Traxler, F Edenhofer, J Martens (2019) Next-generation disease modeling with direct conversion: A new path to old neurons. FEBS Lett.

42. X Ma, L Kong, S Zhu (2017) Reprogramming cell fates by small molecoles. Protein Cell 8: 328-348.

43. L Wang, Y Su, C Huang, Y Yn, J Zhu, et al. (2019) FOXH1 is regulated by NANOG and LIN28 for early-stage reprogramming. Sci Rep 9: 16443.

44. K Moluku, T Harkness, J Carison-Stevemer, R Prestil, NJ Piscopo, et al. (2019) Tracking and Predicting Human somatic cell reprogramming using nuclear characteristics Byophis J s0006-3495: 30862-30868.

45. ML Good, R Vizcardo, T Maeda, N Tamaoki, P Malekzadeh, et al. (2019) Using human induced pluripotent stem cells for the generation of tumor antigen-specific $T$ cells. $J$ Vis Exp 152. 